\title{
Quality Control and Structural Assessment of Anisotropic Scintillating Crystals
}

\author{
Luigi Montalto ${ }^{1,2, *}$, Pier Paolo Natali ${ }^{3}$, Lorenzo Scalise ${ }^{2,4}\left(\mathbb{0}\right.$, Nicola Paone ${ }^{2,4}$,

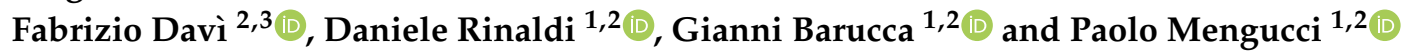 \\ 1 Dipartimento di Scienze e Ingegneria della Materia, dell'Ambiente ed Urbanistica (SIMAU), \\ Università Politecnica delle Marche, 60131 Ancona, Italy \\ 2 Interdipartimental Crystal Research \& Analysis Center (ICRYS), Interdepartmental Crystal Research \& \\ Analysis Center, 60131 Ancona, Italy \\ 3 Dipartimento di Ingegneria Civile, Edile e Architettura (DICEA), Università Politecnica delle Marche, \\ 60131 Ancona, Italy \\ 4 Dipartimento di Ingegneria Industriale e Scienze Matematiche (DIISM), Università Politecnica delle Marche, \\ 60131 Ancona, Italy \\ * Correspondence: 1.montalto@univpm.it
}

Received: 29 June 2019; Accepted: 15 July 2019; Published: 23 July 2019

\begin{abstract}
Nowadays, radiation detectors based on scintillating crystals are used in many different fields of science like medicine, aerospace, high-energy physics, and security. The scintillating crystals are the core elements of these devices; by converting high-energy radiation into visible photons, they produce optical signals that can be detected and analyzed. Structural and surface conditions, defects, and residual stress states play a crucial role in their operating performance in terms of light production, transport, and extraction. Industrial production of such crystalline materials is a complex process that requires sensing, in-line and off-line, for material characterization and process control to properly tune the production parameters. Indeed, the scintillators' quality must be accurately assessed during their manufacture in order to prevent malfunction and failures at each level of the chain, optimizing the production and utilization costs. This paper presents an overview of the techniques used, at various stages, across the crystal production process, to assess the quality and structural condition of anisotropic scintillating crystals. Different inspection techniques (XRD, SEM, EDX, and TEM) and the non-invasive photoelasticity-based methods for residual stress detection, such as laser conoscopy and sphenoscopy, are presented. The use of XRD, SEM, EDX, and TEM analytical methods offers detailed structural and morphological information. Conoscopy and sphenoscopy offer the advantages of fast and non-invasive measurement suitable for the inspection of the whole crystal quality. These techniques, based on different measurement methods and models, provide different information that can be cross-correlated to obtain a complete characterization of the scintillating crystals. Inspection methods will be analyzed and compared to the present state of the art.
\end{abstract}

Keywords: quality control; photoelasticity; residual stress; crystals defects; non-destructive techniques; measurement; scintillating crystals; structural assessment; fast inspection; online measurements; inspection systems

\section{Introduction}

Scintillating crystal-based detectors are the main devices used for radiation and particle detection. The scintillating substances, by converting radiation into visible light, produce the main signal, which is then acquired by photoelectric sensors and analyzed by electronic and computing devices. Thanks to this capability, they are key components in a variety of measurement systems and diagnostic 
machines and are relevant in a number of scientific and technical areas whose impact on society is great: medicine, aerospace, security, and high-energy physics are some of the fields in which scintillators are involved [1,2]. These challenging environments are continuously pushing research and industry towards better-performing systems in order to meet the increasingly demanding requirements of their fields [3,4]. Medical devices for cancer diagnosis and treatment as well as for nuclear imaging (PET, SPECT, and CT scanner systems) require spatial resolution, accuracy, and fast response in order to achieve efficient diagnosis and treatment of patients at a reduced cost and time of exposure. The $10 \mathrm{ps}$ coincidence time resolution (CTR) challenge for PET systems aims to produce a real-time, detailed medical imager for fast and minimally invasive procedures [5,6]. Environmental scanners need energy resolution and sensitivity in order to be able to discriminate the amount and type of radiation. Security systems must be fast and sensitive in order to reduce the dose and the time for an accurate inspection of the suspicious substances. Systems for aerospace and high-energy physics instruments need reliability, durability, and stability over a wide range of conditions and time. For example, calorimeter mechanical design imposes project specifications on the ultimate tensile strength and elastic properties of crystals [7,8]. Scintillators must withstand severe environmental demands in terms of temperature, mechanical stress, moisture, and radiation dose. Some examples are the high-luminosity LHC project and the new PANDA and Mu2e projects [9,10], in which the scintillating crystals must maintain light transport and collection for as long as possible under a severe radiation regime and uncontrollable environmental conditions. Scintillators are found in industry, exploited in detectors aimed at process and material testing, and in scientific devices too (e.g., X-ray diffractometers) which play a role in control, inspection, and investigation. Monocrystalline scintillators are still the most efficient and promising material to meet the new challenging requirements and future applications [3]. Among them, anisotropic ones like PWO, LYSO, ZnWO, and LaBr are the most reliable and accurate [11,12].

The production process of such materials and their tailoring for different purposes are complex and expensive. These crystals are grown by basic two methods, Bridgman and Czochralsk; both involve high-temperature furnaces in which the raw materials are melted; subsequently, the melted substance is put in contact with a high-quality crystalline seed and slowly cooled. The control of atmosphere, gaseous pressure, temperature, and speed are critical in order to obtain stable crystal growth, in which the condition for the substance to organize itself into a monocrystalline structure with a homogeneous distribution of the possible dopants is met. The crystal "boule" (the unrefined bulk crystal) is then finished and worked according to the intended tasks; it is usually cut into different dimensions and geometries according to the detector system's specification. Surface treatments and, possibly, special coatings can be applied in order to enhance the light transport and collection properties. Overall, crystal production is a multi-stage process for which process control and quality control play a fundamental role in preventing defects.

Although anisotropic crystals offer very high performance, they are very sensitive to stress, structural conditions, and defects $[13,14]$. Small changes in the production parameters or environmental conditions can create unwanted defects that dramatically affect the scintillating behavior, reducing the crystals performance and lifetime. Since they are brittle materials, the residual stress must be taken into account to prevent unwanted mechanical failures (even during the production process) and internal fractures that make those materials unusable [15]. Structural macro-distortions, dispersion of inclusions and/or impurities, and dislocation distribution play a crucial role in the scintillator light production, transport, and collection. The collection of the visible photons produced by the scintillator is the signal transmitting information on the observed phenomena; therefore, the surface texture and condition are crucial in order to have a perfect match with the photosensor.

Quality control and monitoring of these features are fundamental at each level of the scintillating crystal's life cycle: research, industry, production, and application. In most cases the quality control of these materials can be attained by visual inspection based on the experience of the production personnel, at the production stage, and by the measurements of the crystal's scintillating and light performances at the end-user level. 
The measurement methods presented in this work give detailed information about structural quality at different scales. Photoelasticity-based techniques $[16,17]$ will be presented in the two different and complementary forms of laser conoscopy and sphenoscopy; these non-invasive techniques, and the associated theoretical models, allow us to collect information about residual stress, macro-defects and structural macro-distortion distribution, producing feedback for the tuning of the production parameters, ensuring the crystals' correct light transport capability and predicting the functional behavior in a fast but reliable manner. These methods are particularly suitable for crystal producers, researchers, and end-users since they are non-destructive and all the samples of the production can be inspected.

As far as extremely detailed inspection and quality assessment methods are concerned, XRD, SEM, EDX, and TEM analytical methods will be surveyed. These techniques give information on the crystal lattice structure, compound distribution, and structural morphology at the micro- and nanometric scale. On the other hand, they require carefully prepared samples, they can be destructive techniques, and they are time consuming since they need special preparation of the sample and complex instrumentation. Finally, we will describe and review the grazing incident diffractometry (GID) method for surface roughness and crystallinity assessment. Some important information about crystals' interfaces can be obtained by this last method. They allow the proper tuning of the finishing procedures aimed at achieving optimal light guidance and collection of the detector system. All these presented methods can be combined to arrive at a qualification procedure for the assessment of the crystals' condition and the qualification of the production process.

\section{Photoelasticity-Based Methods}

Photoelasticity has been used for many decades for assessing the stress condition in isotropic and transparent materials like glass and Perspex; in some cases these materials are arranged in geometries similar to the mechanical and structural systems whose load-induced stress distribution have to be investigated $[7,16,17]$. Photoelasticity can be implemented in several modes (e.g., transmission, reflection, and scattered modality); in all cases, photoelasticity generates fringe patterns that depend on the state of stress of the analyzed structure [16,17]. No fringes are generated in isotropic media if no stress or no refraction index variation are present into the volume. On the contrary, in anisotropic crystals, fringe patterns are present even in unstressed conditions due to their crystallographic structure. This led to more complex methodologies aimed at carrying out and analyzing the fringe patterns' shape as a signature of defects and stress. In the following, we describe some recently developed methods that merge photoelasticity and optics for anisotropic crystal inspection.

\subsection{Laser Conoscopy}

\subsubsection{Conoscopic Fringe Patterns and Their Interpretation}

Conoscopy is an optical crystallographic technique that uses polarized light to acquire information about the crystalline materials and their optical characteristics. It is classically implemented in polarized microscopes for observing thinly sliced samples for mineralogy purposes [18]. The technique has been adapted to measure residual stress in anisotropic crystals (a synonym for naturally birefringent crystals).

The crystal optical properties are described by the inverse permittivity or dielectric impermeability tensor $\boldsymbol{B}$, a second-order symmetric tensor whose principal components $B_{x}, B_{y}$, and $B_{z}$ are associated with the principal refractive indices $n_{x}, n_{y}$, and $n_{z}$ by:

$$
B_{i}=\left(\frac{1}{n_{i}}\right)^{2} B_{i}=1 / n_{i}^{2}, i=x, y, z
$$

The tensor $\boldsymbol{B}$ is associated to the optical indicatrix or index ellipsoid (Figure 1a), whose equation is

$$
B X \cdot X=1 ; X=(x, y, z)
$$


which is a sphere for isotropic crystals (three equal refractive indices), a rotation ellipsoid (spheroid) for a uniaxial crystals (two equal refraction indices with one optic axis) or a general ellipsoid for biaxial crystals (three different refraction indices and two optic axis that are orthogonal to the planes that intersect the ellipsoid into a circumference). The light crossing the crystal's volume is affected by the refraction indices; its velocity and polarization are changed as a function of the index ellipsoid (Figure 1b) $[19,20]$. In a plane polariscope configuration, each polarized ray of light that impinges on the crystal surface is split and orthogonally polarized. They exhibit a phase delay and an interference pattern is obtained when they are recombined after the analyzer (Figure 1b,c).

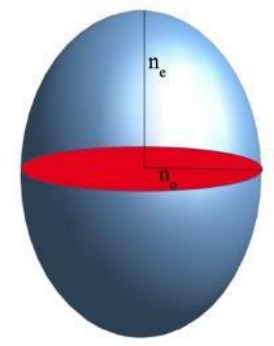

(a)

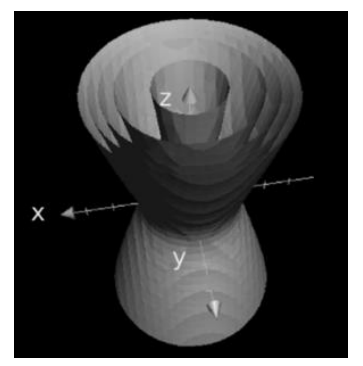

(d)

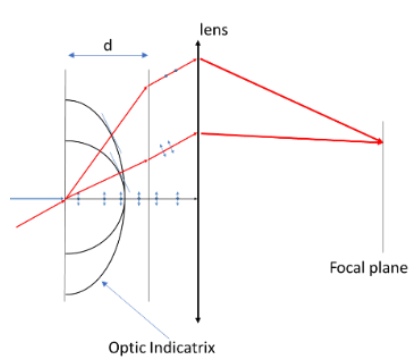

(b)

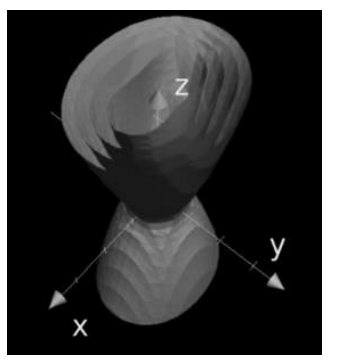

(e)

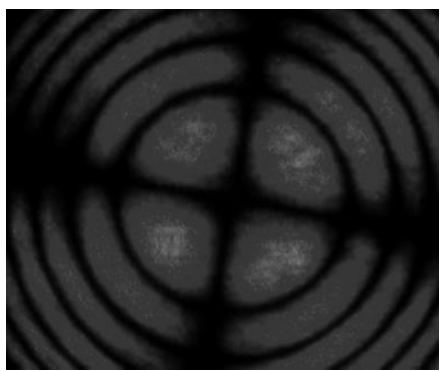

(c)

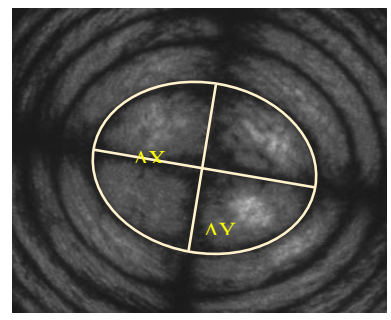

(f)

Figure 1. (a) The optic indicatrix of a uniaxial crystal. The semi-axes of the ellipsoid (prolate or oblate) are the refraction indices. The two refraction indices $\left(n_{o}\right.$ and $\left.n_{e}\right)$ are shown. In biaxial crystals there are three refraction indices as the optic indicatrix is a general ellipsoid. (b) A $2 \mathrm{~d}$ scheme of the birefringent phenomenon under which the light goes crossing a birefringent sample with thickness d; the double-arrowed lines and the dots represent two orthogonal polarizations of light. (c) Conoscopic fringe pattern observing the sample along its optic axis. (d) A simulation of the Bertin iso-delay surfaces; the optic axis is the $z$ axis. (e) Simulation of Bertin surfaces for a stressed uniaxial crystal and/or a naturally biaxial crystal, where the $z$ axis is the optic axes bisector. (f) The fringe pattern obtained by observing a stressed uniaxial crystal or, possibly, a biaxial crystal observed along the acute bisector of the optic axes (the $z$ axis in (e)) (used with permission from Copyright Clearance Center/AIP Publishing, [21]).

As an example, in Figure 1c we show a typical interference image for a uniaxial crystal observed along its optic axis. The fringe orders are due to the optical path difference according to:

$$
N \lambda=\frac{d}{\cos \theta}\left(n_{e}-n_{0}\right) \sin ^{2} \theta,
$$

where $N$ is the fringe order (or the delay), $\lambda$ is the wavelength of the light, $n_{0}$ and $n_{e}$ are the ordinary and extraordinary refractive index, respectively, $\theta$ is the angle between the ray of light and the optic axis, and $d$ is the sample thickness. Equation (3) holds for uniaxial crystals; otherwise, in biaxial ones, the Fresnell law changes to:

$$
\Delta=\frac{2 \pi}{\lambda} \rho\left(n_{x}-n_{z}\right) \sin \theta_{1} \sin \theta_{2}
$$


where $\rho$ is the mean ray between the ordinary and the extraordinary one, $\lambda$ is the wavelength of the impinging light, $\theta_{1}, \theta_{2}$ are the angles formed by the ray and optic axes, respectively, and $\Delta$ the phase difference between the ordinary and extraordinary rays.

These patterns are due to the structure of the crystals and are based on the theory of the Bertin surfaces [22-24] (Equation (2)), which are the virtual iso-delay surfaces that the light generates when crossing the sample (Figure 1d,e). As a matter of fact, Bertin's surfaces are the bijective relation between the light direction angle with the optic axes and a point in space and can be expressed as $[19,20]$ :

$$
(N \lambda)^{2}=\left(x^{2}+y^{2}+z^{2}\right) \cdot\left(n_{x}-n_{z}\right)^{2}\left(1-\frac{[z \cdot \cos \beta+x \cdot \sin \beta]^{2}}{x^{2}+y^{2}+z^{2}}\right) \cdot\left(1-\frac{[z \cdot \cos \beta-x \cdot \sin \beta]^{2}}{x^{2}+y^{2}+z^{2}}\right) .
$$

Equation (5) is the general expression of the Bertin's iso-delay surfaces in Figure 1d,e. The angle $\beta$ is the angle between the optic axes and their bisector (the $z$ axis in Figure $1 \mathrm{e}, \mathrm{d} ; \beta=0$ in uniaxial crystals). The $\beta$ angle of the acute optic bisector $(O B)$ is given by:

$$
\operatorname{tg}^{2} \beta=\frac{\left(\frac{1}{n_{y}}\right)^{2}-\left(\frac{1}{n_{x}}\right)^{2}}{\left(\frac{1}{n_{z}}\right)^{2}-\left(\frac{1}{n_{y}}\right)^{2}}
$$

The interferograms in Figure 1c,f can be modeled as the intersection of the surfaces in Figure 1d,e with a plane orthogonal to the $Z$ axis. Whenever a stress is applied on the crystal, the Bertin surfaces and, consequently, the fringe pattern deforms (Figure 1e,f). The Bertin surface distortion depends on the fourth-order piezo-optic tensor $\pi$, which depends on the crystal symmetries, as follows:

$$
B(\mathrm{~T})=B_{0}+\pi[\mathrm{T}]
$$

where $\boldsymbol{B}_{\mathbf{0}}$ is the dielectric impermeability tensor in unstressed condition; a detailed description of the related theory is given in [25].

Due to the anisotropy, the generated patterns are a function of the observation direction with respect to the $O B$ (Figure 1c,f and Figure 2a,b).

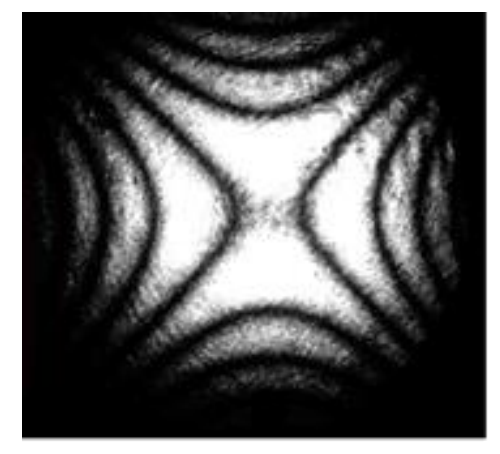

(a)

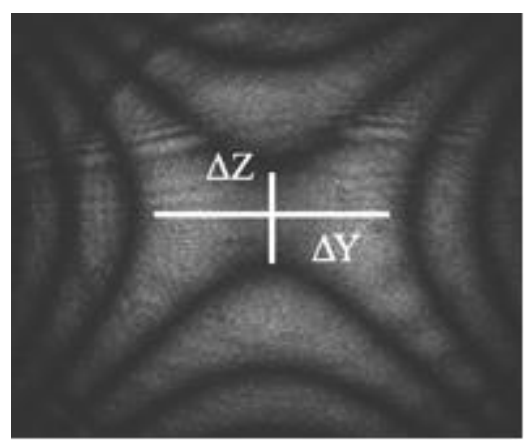

(b)

Figure 2. (a) Fringe pattern obtained observing a uniaxial crystal sample orthogonally to the optic axis (the $X$ or $Y$ axis in Figure 1d) or a biaxial sample along its obtuse bisector (the $X$ axis in Figure 1e).

(b) The measurement method for achieving the stress measurement, $(\Delta \mathrm{Y}-\Delta \mathrm{Z})=k_{0}+k_{\sigma} \sigma$.

In this paper, we concentrate on the two main directions in uniaxial crystals, but the method and the technique can be extended to biaxial ones. The fringe pattern obtained observing along the $\mathrm{OB}$ (optic axis in unstressed uniaxial crystals) deforms in case of stress. The circular fringe orders (in unstressed uniaxial crystals) alter into fourth-order Cassini Like (CL) curves that approximate ellipses 
for small stress. Measuring the major and minor axis of the elliptic first fringe order, the stress can be evaluated via

$$
\sigma=\frac{C}{f_{\sigma}}
$$

where $f_{\sigma}$ is the photoelastic constant and $C$ is the ellipticity measured, referring to Figure $1 \mathrm{f}$, following the semi-phenomenological relation:

$$
C_{\sigma}=\frac{\Delta X}{\Delta Y}-1=f(\sigma, \pi),
$$

where $\Delta X>\Delta Y$ always holds true in reference to Bertin surfaces (Figure 1d,e) [25].

Once the uniaxial crystal is stressed, it behaves like a biaxial crystal observed along the acute bisector of its optic axes, so the method can be extended to this latter category. In fact, Equation (9) is also valid for naturally birefringent crystals, taking into account that the unstressed interference pattern is composed of CL curves. For the analytical expression of the photoelastic constant and the ellipticity, refer to [25-27]. By observing orthogonally to the optic axis (or along the obtuse bisector of biaxial crystals), the interferogram appears as a series of hyperbolas (Figure 2a,b). In this case, the distance between the hyperbolas of the branches is linked to the stress condition by the following relation (Equation (10)), which is derived from a more complex model [14]:

$$
R=(\Delta-\Delta Y)=k_{0}+k_{\sigma} \sigma,
$$

where $k_{0}$ is the difference $R$ in unstressed condition and $k_{\sigma}$ is the photoelastic constant relevant to this direction.

If the piezo-optic tensor $\pi$ elements are unknown, in both observation directions a calibration step is required in order to evaluate the photoelastic constants. Finally, these methods allow us to evaluate the internal stress state, which is linked to the whole crystal quality.

Interference and conoscopic methods are also useful to evaluate the piezo-optic surface, stress, and the $\pi$ tensor elements [28-30]. That method is based on the difference between the radius vector in the optic indicatrix perturbed by stress and the unstressed one.

\subsubsection{Implementation}

Laser conoscopy is based on the plane/circular polariscope setup [21,31,32]. Despite its reliability, the classical diffused light source has been substituted with a polarized laser source. In fact, diffused light polariscopes have limitations in terms of their spatial resolution, sensitivity, and complexity of analysis when used for anisotropic media. Since the sample is fully illuminated, the pattern carried out by the diffused light polariscope is due to the whole volume; therefore, complex shapes are obtained if the stress distribution is not uniform over the sample [22]. Moreover, this full field technique has a limited sensitivity to local variations in the stress condition since they are averaged on the entire crystal volume [32]. Collimated laser light with a special optical layout allows us to confine the light in a known portion of the crystal volume (Figure 3a,b).

As the volume decreases, the sensitivity and spatial resolution increase. In the same manner, the complexity of the fringe pattern is reduced if a stress gradient or complex stress distribution is present over the crystal volume [32]. Since the fringe pattern is generated solely by the illuminated volume, it is reasonable to consider the stress uniform if this volume is small. This leads to the generation of simple geometric shape of the fringes (circles, ellipses, or hyperbolas). The improved spatial resolution of this pointwise method is paid with the necessity of a scanning procedure to map the entire crystal sample in detail. 


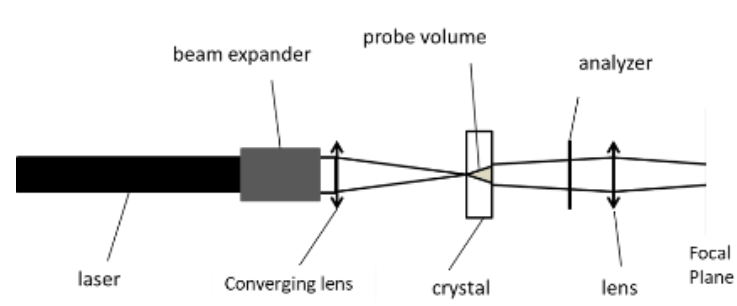

(a)

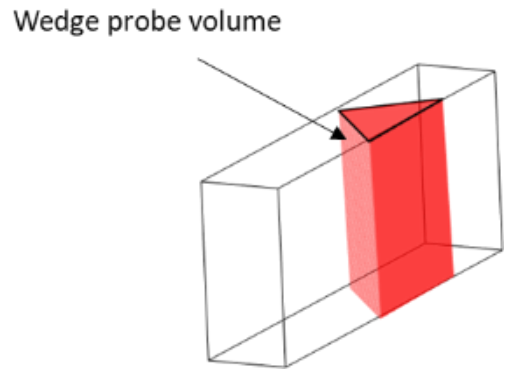

(c)

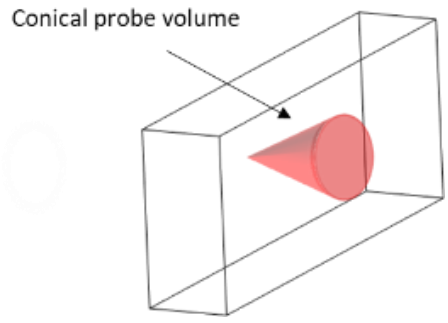

(b)

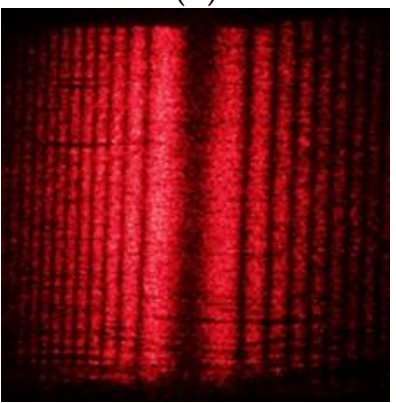

(d)

Figure 3. (a) The basic scheme of the laser conoscopy/sphenoscopy technique. The main difference is the use of a spherical (conoscopy) or cylindrical (sphenoscopy) lens that generates different probe volumes. (b) The conoscopic probe volume for the punctual inspection. (c) The wedge-shaped volume generated by sphenoscopy, which allows us to analyze an entire line of the sample. (d) The sphenoscopic fringe pattern obtained along the optic axis. The stripes are due to the envelope of the conoscopic fringe orders along the height of the wedge (used with permission from Copyright Clearance Center/AIP Publishing, [33]).

\subsection{Sphenoscopy}

Laser conoscopy provides details for each point of observation; however, the technique may require a large number of scanned points to cover an extended sample. Therefore, this procedure may not always be compatible with those scenarios that require a very short testing time (e.g., in line inspection of mass production systems). For this it is possible to introduce a new inspection layout, leading to the new concept of sphenoscopy [33]. The scheme in Figure 3a has been modified, substituting a cylindrical lens for the spherical one. Since the focus is a line instead of a point, the light volume generated in the crystal is a thin wedge (Figure 3c). By this method an entire line is inspected, instead of a series of points, in one single acquisition. Consequently, the inspection procedure could be reduced from $(n \times n)$ acquisitions to $(n+n)$ acquisitions. The sphenoscopic fringe pattern is simplified with respect to the conoscopic one; as with the Bertin model, the light spans over a plane angle only, so one dimension of the fringe orders is obtained. Different to the laser conoscopy, where the iso-delay surfaces are a bijective function of an angle that spans the three spatial dimensions, the sphenoscopy is due to the bijective relation between a plane angle and the space. The pattern is generated by the envelope of this dimension over the light wedge height, which lead to a fringe figure composed of a series of lines (Figure 3d). The presence of defects, stress, or non-uniformities over the inspected volume modifies the lines' curvature and the distances between them. The complexity of the pattern interpretation is, therefore, reduced. Even though the information gathered in this method is less than the conoscopic figures, the results are comparable. Sphenoscopic inspection remains suitable, for instance, for a fast control of conformity.

The methodologies detailed in this paragraph offer a set of possibilities to build a non-invasive and fast control system that is manageable and adaptable to different conditions and scenarios of inspection and monitoring.

Applicability of such methodologies spreads over the entire scintillating crystals value chain. The non-invasiveness and speed of the techniques are particularly useful at the production stage. The 
entire population of crystals can be inspected by photoelastic-based methods. This monitoring can be implemented at different stages of the process: for instance, soon after the growth, to implement growing process evaluation and decide on the best cutting procedure; before and after the machining process to assess the process itself, monitor possible defects, prevent unwanted failures, etc. An inspection of the incoming samples can be easily done by the end-user (such as detector producers and assemblers) to recognize and select samples with a low level of defectiveness. Research bodies have a powerful tool to study the link between structure, defectiveness, and performance of the scintillators. Stress structures, observed by photoelasticity, indicate macro-distortion of the lattices, which can be due to the production process and/or the presence of defects such as unwanted precipitates or clusters of particles that may affect the mechanical properties and the scintillation performances. In fact, the distribution of stress and defects influences the light transport and collection since the crystals can undergo lensing effects, generating unwanted focal points inside the structure and the modification of internal reflection conditions, and creating a preferential light path into the sample volume that can affect properties like decay time, energy resolution, and light yield. Moreover, the presence of such defects can affect the scintillation process itself, for example by establishing a piezo-spectroscopic regime.

\section{SEM, TEM, and EDX Analysis, X-Ray Scattering, and Reflectivity Methodology}

Electron microscopy techniques are largely used in materials science because of their ability to provide microstructural data, indispensable for understanding the macroscopic features of the materials. The comprehension of the connections between microstructure and macro-properties allows for the design and realization of new and better-performing materials. Furthermore, SEM and TEM microscopes are usually equipped with an EDX microanalysis system able to locally investigate the chemical composition of the sample. Concerning scintillating crystals, the following aspects can be investigated by electron microscopy techniques.

The presence of defects, rugosity, and inhomogeneities, both artificially made and intrinsically present, on the surface of a crystal can be relevant for determining the quantity of light that can be collected, and thus the sensitivity and the efficiency of the detection system. These features may have a size on the order of some nanometers and can be easily investigated by scanning electron microscopy techniques (SEM). Indeed, this technique allows us to analyze the surficial morphology of a sample in a large range of dimensions (from $\mathrm{cm}$ to $\mathrm{nm}$ ) using secondary electrons. On the other hand, backscattered electrons are used in SEM to obtain information on possible surficial composition inhomogeneities. This can be carried out with greater accuracy by using an EDX microanalysis system. This system is typically associated with an electron microscope. In EDX spectroscopy, the energy of characteristic X-rays emitted during the interaction of the electron beam with the sample is analyzed to identify the chemical element present in the sample. Due to the small dimension of the electron beam, the chemical analysis can be performed on volumes of a few $\mu \mathrm{m}^{3}$.

In order to investigate the inner structure of the crystals at atomic resolution, transmission electron microscopy (TEM) techniques are very powerful. By TEM it is possible to investigate the presence of lattice defects (precipitates, dislocations, twins, etc.) that could be detrimental to light emission. The crystallographic structure of the sample can be investigated, and the anisotropic axis determined. The composition of the sample can be analyzed by EDX spectroscopy at a spatial resolution of few nanometers or even lower, by using a TEM electron beam.

Some sample preparation must be done before electron microscopy observations, however. The typical requirement for samples to be observed by SEM is that they must be conductive. This is easily obtained for non-conductive materials, by covering them with a very thin film of metal or carbon; the latest microscopes allow one to set the electron beam energy to such low values that, in some cases, it is possible to perform SEM observations on non-conductive materials without any conductive film over them. More restrictive are the requirements for TEM samples: they must be so thin as to be transparent to the electron beam (typically $\leq 100 \mathrm{~nm}$ ) and cannot be larger than $3 \mathrm{~mm}$ in diameter. 
To obtain samples with these specifications requires very sophisticated and quite expensive (both in terms of money and, mostly, time) techniques.

Investigation techniques based on X-ray scattering are largely used for material characterization at the nanoscale [34-36]. X-rays are electromagnetic waves with a wavelength of around one angstrom $\left(10^{-10} \mathrm{~m}\right)$, hence particularly useful for investigating structures such as atoms, molecules, and crystals. As $\mathrm{X}$-rays are scattered by single electrons, atoms, molecules, and crystals, penetration inside matter depends on the material density as well as on the angle $(\theta)$ that the incident $X$-ray beam forms with the sample surface. At grazing incidence $\left(\theta=0.4^{\circ}-5^{\circ}\right)$, penetration is limited to near-surface regions so that the surface structure of materials can be easily investigated.

For detectors based on scintillating crystals, surface roughness and defects strongly limit the optical performance of the system.

Grazing incidence X-ray diffraction (GID) can be successfully used to investigate the crystalline state of surface or near-surface regions, compositional depth profile, presence of residual stresses, or crystallographic defects due to the growth process or the surface finishing post-production processes [13].

To investigate surface roughness, material density and the presence of a surface layer or multilayer systems X-ray reflectivity (XRR) is commonly used. As XRR is based on radiation reflection at surfaces and interfaces, the technique estimates the roughness, thickness, and density of a single layer or multiple layers independently of sample crystallinity.

On the other hand, investigation of the material inner volume is performed by $\mathrm{X}$-ray diffraction (XRD) techniques at higher incidence angles $\left(\theta=5-60^{\circ}\right)$. Material density determines the size of the analyzed volume from which information is averaged. In this sense, XRD is considered a volume technique, in contrast to microscopic techniques (SEM, TEM) $[37,38]$ that show lateral resolution at the nanoscale. As XRD is based on the radiation reflection on crystalline planes of the material, only crystalline materials (single crystals or polycrystals) can be successfully investigated in terms of compound information, lattice parameters, crystallography, and residual stresses. Analysis of the inner structure of amorphous materials or short-range ordered materialsat the nanoscale is always limited.

XRD measurements of scintillating single crystals are used to determine the exact orientation of crystallographic axes, which is correlated to optical performance.

The widespread use of X-ray-based analytical techniques (XRD, GID, XRR) in the field of scintillating crystals is due to the fast sample preparation and measurement times. For scintillating crystals with a size comparable to or below the centimeter range, sample preparation is practically unnecessary, while measurement times can vary from minutes to a few hours depending on the X-ray facility. Data interpretation can be difficult in the case of a highly defective structure or a combination of disordered crystallographic compounds.

As a general rule, complete characterization of scintillating crystals at the nanoscale requires two or more complementary analytical techniques, such as electron microscopy and X-ray scattering.

\section{Example of the Use of the Presented Measurement Techniques}

Each technique mentioned provides structural information at a different scale. Depending on the features and dimension of details to be detected, a suitable methodology can be chosen. On the other hand, by using several different and complementary analytical techniques, a more accurate and complete characterization of crystal quality can be achieved. Moreover, the latter procedure helps with understanding the correlations between different features and phenomena within the sample structures and its behavior.

The non-invasiveness and fast nature of the photoelasticity-based methods have been exploited for the quality assessment and selection of PWO crystals for the CMS experiment at CERN [15]. A pre-production series of PWO growth by the Bridgman technique [39] has been inspected by conoscopy. Observations allowed for evaluating the residual stress, due to the production process, as a signature of the whole quality of both the crystals and their manufacturing procedure. The 
residual stress state is an indication of a correct production process, from growth to machining and final finishing. This is linked to the general quality of the scintillators. Scintillation can be affected, and unwanted failures may arise even due to the interaction with heavy particles like hadrons. Two ingots were cut into slices and inspected in different positions. Since the observations were performed along the optic axis, the evaluation of the residual stress was carried out by the detection of the ellipticity. Figure 4a schematically reports the ellipticities of six points over the surface of six slices. In the graph in Figure $4 b$, the averaged residual stress in different positions is reported. The photoelastic method has allowed the selection of higher-quality samples and has given indications for tuning the growth parameters properly, thus enhancing the production yield. In that work [15] a quality index has been proposed for the selection of suitable crystals (Figure $4 \mathrm{c}$ ). That index is defined as $R=\left[(k S)^{2}+\sigma_{a v}^{2}\right]^{1 / 2}$, where $\sigma_{a v}$ is the average value of stress, while $S$ is the standard deviation. The coefficient $\mathrm{k} \geq 1$ is related to the producer experience, which weights the dispersion of the data.

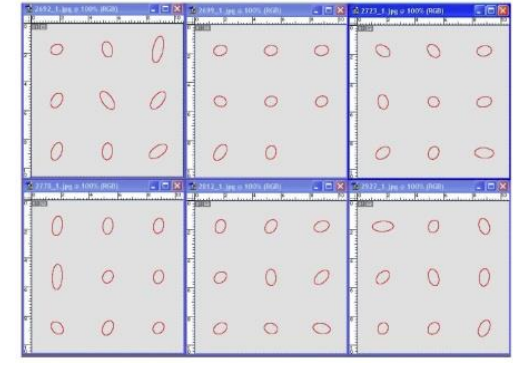

(a)

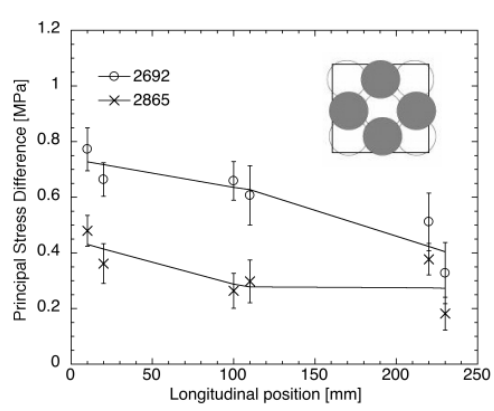

(b)

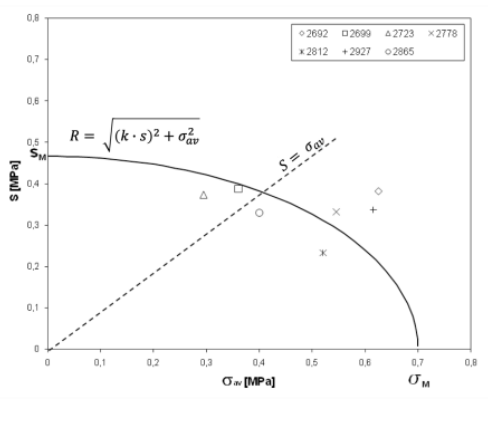

(c)

Figure 4. (a) The ellipticities evaluated by conoscopy on six cross sections. (b) The average residual stress measured at different positions along the length of the samples. (c) By the measurement of the residual stress, an indication of the whole quality can be achieved; this schematically represents the region of quality acceptance following the index reported on the graph (used with permission from Copyright Clearance Center/Elsevier, [39]).

In PWOs for the CMS experiment, the influence of surface finishing has been also studied, exploiting the GID analysis [40]. Samples were prepared and treated by different surface finishing techniques. GID analysis was used to investigate the surface structure of PWO single crystals submitted to two different finishing processes, mechanical diamond polishing (MDP) and chemical mechanical polishing. GID has clearly shown that CMP tends to amorphize the crystal surface, while the surface crystallinity is maintained when the MDP finishing process is adopted (Figure 5). The results gave a clear indication of how to choose the optimal surface treatment for improving crystal light collection. In the same paper, the results of GID analysis and light yield test were compared, highlighting the positive contribution of the CMP treatment to the optical performance of crystals (Table 1 in Figure 5) [13]. In fact, CMP generates an amorphized but smoother surface that contributes to an enhanced light yield.

A fruitful combination of techniques can be found in a recent paper [14] on modern PWOs (faster and with enhanced light yield). In that work laser conoscopy and sphenoscopy were used to inspect a defective PWO crystal as a pre-series sample for the PANDA experiment in FAIR [9,41]; the assessment of the quality was completed by XRD and EDS analysis, obtaining structural and compositional information. Since the sample was cut along the a-c crystallographic plane, the fringe patterns for each point of inspection were similar to Figure 2. A map of relative stress distribution has been achieved by the conoscopic and sphenoscopic techniques (Figure 6b). In Figure 6a the conoscopic and sphenoscopic images are reported one beside the other.

Patterns shown in Figure 7a were obtained from the XRD analysis, providing information on crystallinity and lattice parameters that are reported in Table 2 in Figure 7 (bottom). Table 2 in Figure 7 
also shows the results of EDS analysis, where the $\mathrm{Pb}, \mathrm{W}$, and $\mathrm{O}$ atomic concentrations are reported as average values (AV) and standard deviations (SD). XRD analysis and conoscopy are in agreement on the angular misalignment between crystallographic plane and sample surface. In particular, the XRD measurement in rocking curve mode (inset in Figure 7) estimated an angular shift of the PWO (200) lattice planes of $4^{\circ}$ with respect to the expected position. During conoscopy measurements, to obtain a symmetric fringe pattern (as in Figure 2), the sample was tilted to the same angular value $\left(4^{\circ}\right)$ from the observation direction.
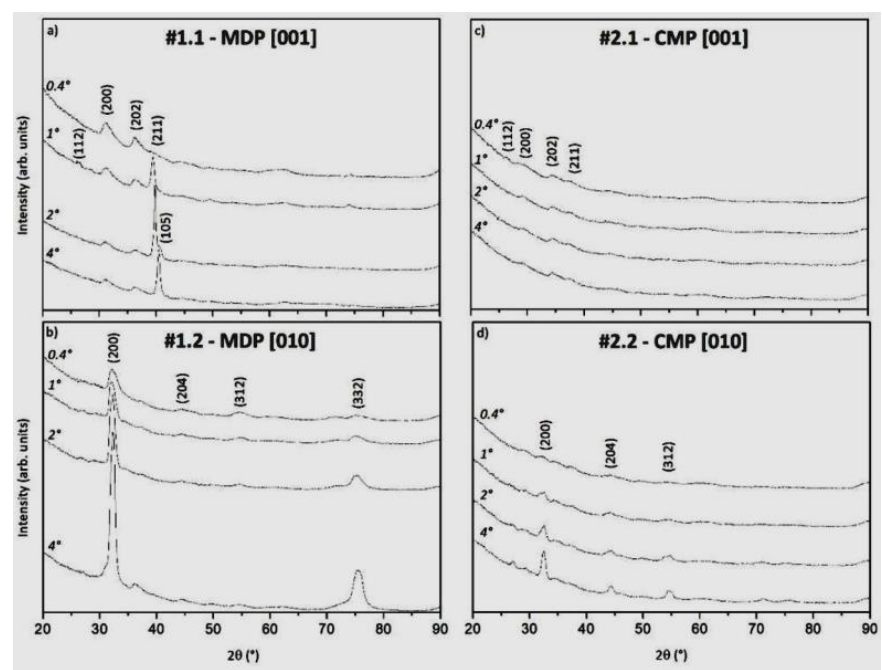

\begin{tabular}{cccc}
\hline $\begin{array}{c}\text { Sample } \\
\text { Number }\end{array}$ & $\begin{array}{c}\text { Surface } \\
\text { Treatment }\end{array}$ & \multicolumn{2}{c}{$\begin{array}{c}\text { Light Yield } \\
\text { (Ph/MeV) }\end{array}$} \\
\hline & & Mean & $\sigma$ \\
& MDP & 12.18 & 0.07 \\
$\# 38138$ & & & \\
& CMP & 12.28 & 0.06 \\
& & & \\
& MDP & 13.40 & 0.13 \\
& & & \\
& & & \\
& CMP & 13.69 & 0.07 \\
& & & \\
\hline
\end{tabular}

Figure 5. GID patterns (left side) at four different incidence angles $\left(0.4^{\circ}, 1^{\circ}, 2^{\circ}\right.$, and $\left.4^{\circ}\right)$ for PWO crystals submitted to MDP and CMP surface finishing. For each treatment, GID analysis was carried out on [001] and [010] crystallographically oriented surfaces. As clearly shown from the GID patterns, MDP maintains the PWO tetragonal structure on the surface, while CMP tends to amorphize the surface. Table 1 (right side) reports results of the light yield test, highlighting the positive contribution of CMP (chemical mechanical polishing) to crystal optical performance with respect to MDP (mechanical diamond polishing).

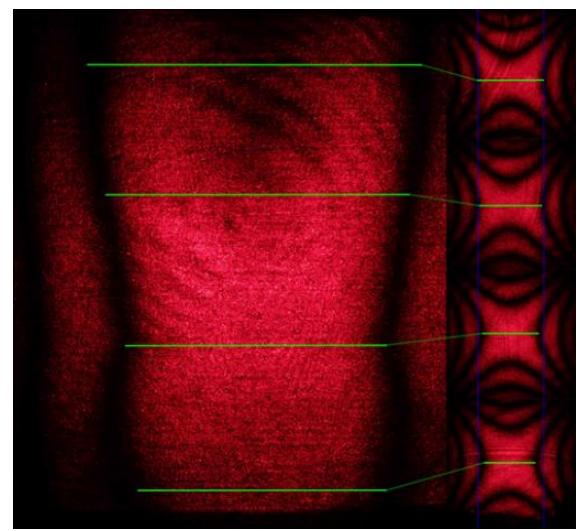

(a)

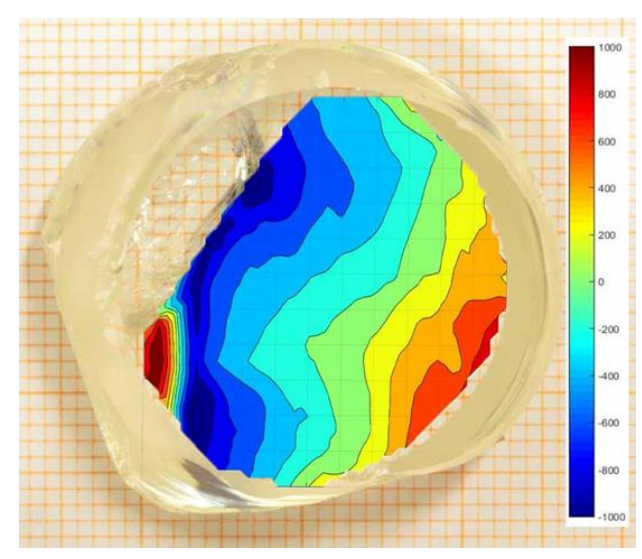

(b)

Figure 6. (a) PWO sphenoscopic image. The inset on the right side shows the conoscopic images carried out over four points, while the larger image represents the sphenoscopic fringe pattern obtained in the same area by a single acquisition [14]. (b) The map of stress distribution superimposed to the sample surface, which is in line with the presence of defects in the PWO crystal [14] (used with permission from IOP; [14]). 


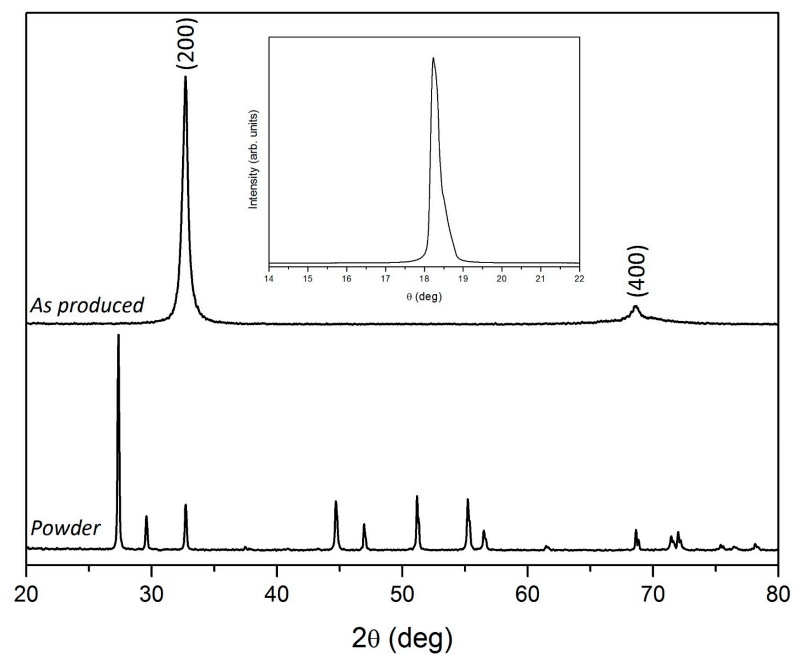

\begin{tabular}{ccccccc}
\hline Compound & \multicolumn{2}{c}{ Pb (at.\%) } & \multicolumn{2}{c}{ W (at.\%) } & \multicolumn{2}{c}{ O (at.\%) } \\
\hline & Mean & SD & Mean & SD & Mean & SD \\
\hline Sample & 20.73 & 1.04 & 21.87 & 1.21 & 57.3 & 2.2 \\
Nominal & 16.7 & & 16.7 & & 66.7 &
\end{tabular}

Lattice parameters (tetragonal structure revealed by $\mathrm{XRD}$ )

$a=0.54676 \pm 0.00015 \mathrm{~nm}$

$c=1.2064 \pm 0.0004 \mathrm{~nm}$

Figure 7. XRD patterns (left) from as produced sample and powder, which was obtained by grinding a small piece of crystal. From peak angular position and relative intensity, PWO crystallographic structure and lattice parameters were calculated. Inset shows the rocking curve of the (200) peak, which reveals misalignment of this lattice plane to the sample surface. The associated Table 2 (right side, top) reports the PWO elements' atomic concentration as a mean value and standard deviation (SD), evidencing slight variations from the nominal values. Table 2 (right side, bottom) reports the lattice parameters of the PWO tetragonal structure.

Techniques like XRD, SEM, and TEM allowed for correlating mechanical properties to the structural characteristics of cerium-doped lutetium yttrium oxyorthosilicate crystals $\left(\mathrm{Lu}_{2\left(1 \_x\right)} \mathrm{Y}_{2 \mathrm{x}} \mathrm{SiO}_{5}\right.$ :Ce namely LYSO:Ce) for medical imaging devices [42-44] in [45]. Ultimate tensile stress (UTS) and Young's modulus (YM) were measured in $[7,46]$. Five of them were correlated to their structural and compositional condition [45]. Table 3, along with Figure 8, shows the UTS and YM values of samples. XRD analysis of samples provided the patterns reported in Figure 8. Rietveld analysis of the XRD patterns allowed for calculating the lattice parameters, which were in close agreement with the literature data.

\begin{tabular}{ccc}
\hline SAMPLE & UTS (MPa) & YM (MPa) \\
\hline$\# 6$ & 68 & 129 \\
$\# 8$ & 78 & 174 \\
$\# 10$ & 79 & 174 \\
$\# 1$ & 94 & 182 \\
$\# 11$ & 114 & 186
\end{tabular}

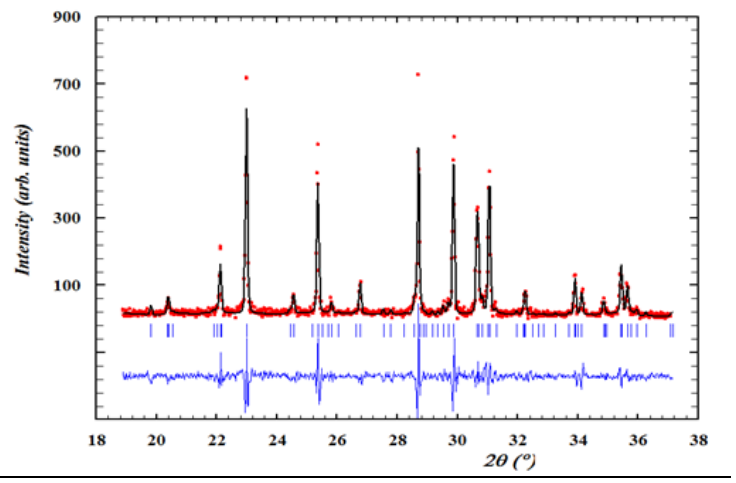

(a)

(b)

Figure 8. The table on the left (a) reports the mechanical properties of the sample, the ultimate tensile stress (UTS), and the Young's modulus (YM). The XRD pattern (b) was obtained from powder. Rietveld analysis confirms the monoclinic structure of LYSO in terms of peak sequence and relative intensity.

By SEM microscopy, surface finishing of samples has been observed. In Figure 9 a typical surface presenting no evidence of macro defects is shown. 


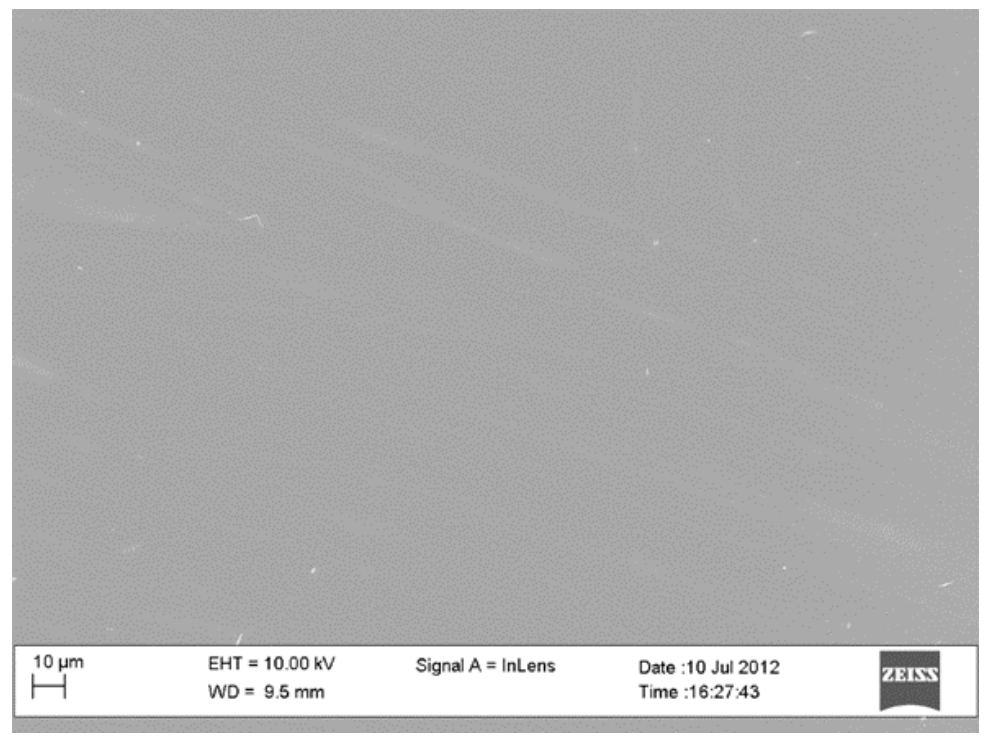

Figure 9. Image of the LYSO crystal surface acquired by a scanning electron microscope (SEM).

TEM measurements were performed on all five samples and the absence of lattice defects, in four out of the five samples, was confirmed by high-resolution analysis (Figure 10a). A completely different microstructure has been revealed in sample \#6, the one with the lowest UTS and YM values.

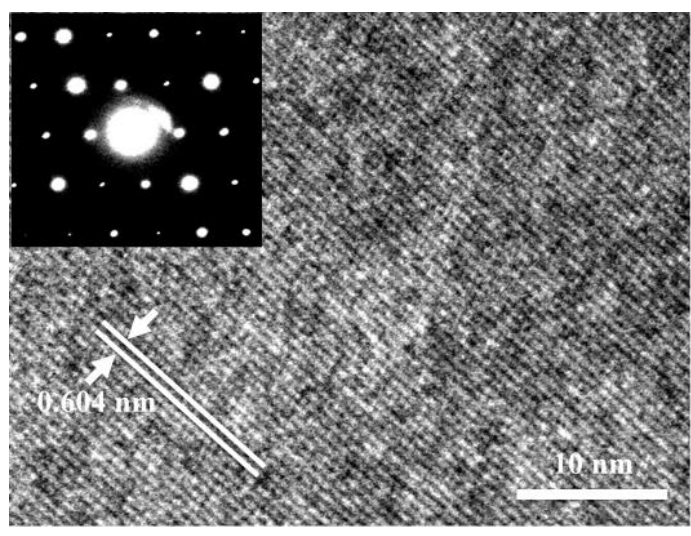

(a)

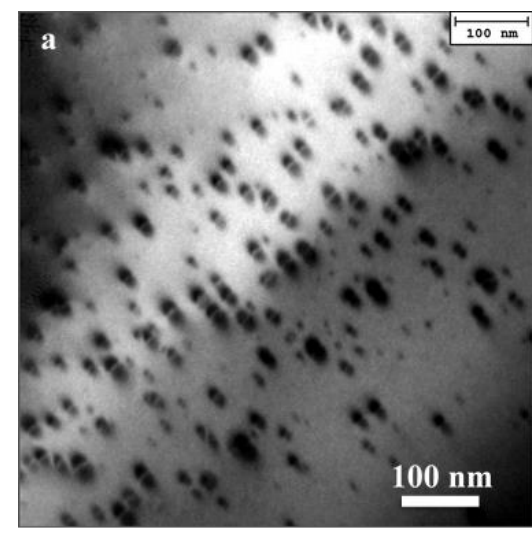

(b)

Figure 10. TEM images. (a) The lattice spacing of the planes is clearly visible and corresponds to the (200) lattice planes of the monoclinic crystal. The inset is the electron diffraction pattern taken in the [010] axis orientation. (b) Area of the sample with a high density of coherent lattice defects.

In fact, despite confirmation of the sample monoclinic structure, a non-homogenous distribution of crystallographic defects has been found. Figure 10b shows a typical "coffee bean" contrast, indicating the presence of coherent particles showing spherically symmetrical strains with the matrix. EDX analysis has revealed an excess of $\mathrm{Lu}$ and $\mathrm{Y}$ in these defective areas, suggesting in the inhomogeneous distribution of these elements the origin of the coherent nanoparticles' formation.

Characterization techniques are crucial for developing new materials to understand which mechanisms rule the behavior of scintillators. In fact, the influence of annealing temperature has been studied by XRD analysis to develop DSB and DSL scintillators [47].

The patterns in Figure 11 highlight how the crystallinity state of the orthorhombic structure varies with a temperature increase in both samples. Therefore, clear indications on the optimization of the production procedure can be achieved from a structural analysis performed by different analytical techniques. 


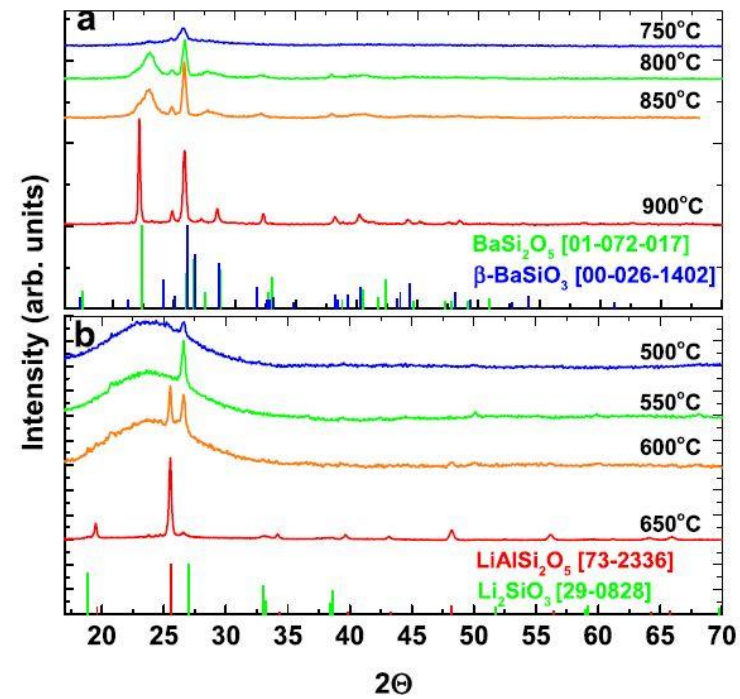

Figure 11. XRD patterns of DSB:Ce (a) and DSL:Ce (b) glasses annealed at different temperatures. Standard reference data are provided, as labeled (used with permission from Copyright Clearance Center/Elsevier, [47]).

The requirements of the application fields are pushing research toward the production of high-quality scintillators shaped like fibers. Their production processes are even more complex than bulky crystals' [48]. The surface to volume ratio of these scintillators is typically extremely high; therefore, the surface condition is critical as well as the crystallinity. Figure 12a shows two SEM images of a LYSO fiber surface produced by the micro-pulling-down (MPD) technique [49]. The surficial roughness can be easily evaluated and measured on large areas of the sample. Due to the dimensions and shape of a LUAG fiber (MDP produced), the sample crystallinity was evaluated by the TEM technique. In particular, TEM analysis revealed that the LUAG fiber is composed of an amorphous matrix with some crystalized nanoparticles (Figure 12b). Due to the shape and dimension of the samples, SEM and TEM techniques are, in these cases, the best possible choice for a structural assessment.

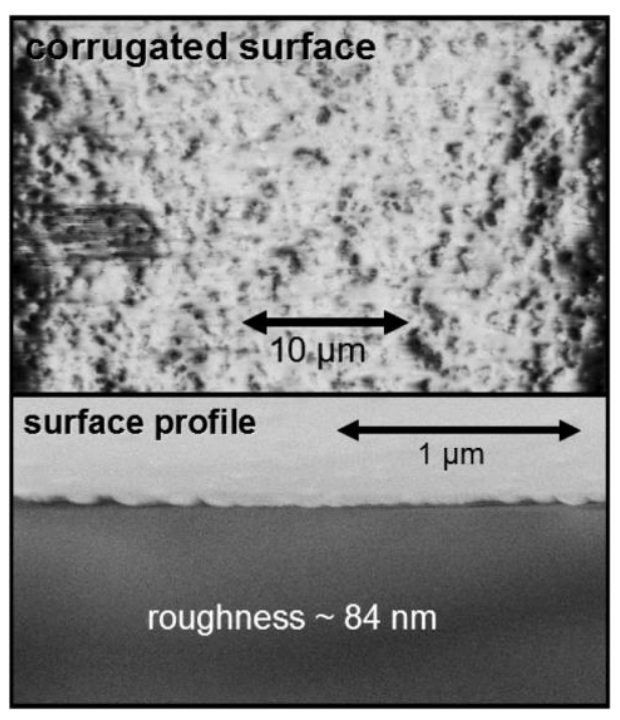

(a)

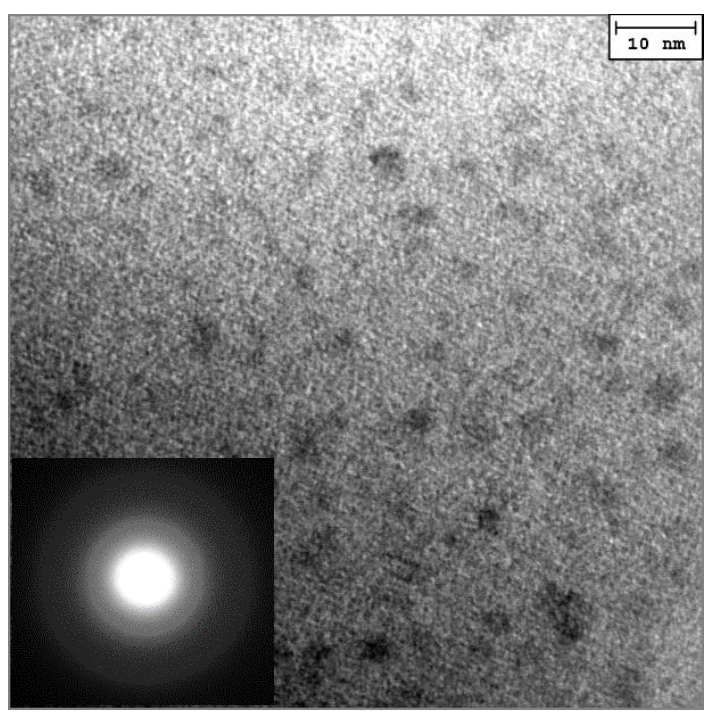

(b)

Figure 12. Scintillating fibers produced by the micro-pulling-down method: (a) SEM images of a LYSO fiber surface; (b) TEM image and corresponding selected area electron diffraction pattern (inset) of a LUAG fiber. 


\section{Conclusions}

The methodologies and measurement techniques presented in this paper provide a wide range of possibilities of exploring and investigating the structural quality of the anisotropic crystals. These techniques may be thought of as complementary to each other; from a macroscopic scale to micro and nanometric features, a complete characterization can be achieved by implementing different methodologies. Innovative laser conoscopy and sphenoscopy are particularly convenient for the quick and non-invasive inspection of crystal macroscopic conditions. Lattice macroscopic distortions due to the presence of residual stress and/or defectiveness can be easily detected. They are particularly helpful in scenarios like the inspection of the complete production of crystals at the grower level and the inspection of incoming samples for detectors assembly; in these situations non-invasiveness and speed are crucial.

More intimate inspections are allowed by the X-ray diffractometry techniques and electron microscopy. In fact, the quality of the lattice and its parameters can be evaluated with accuracy by XRD. This enables us to characterize the crystallinity and the possible lattice distortions due to inclusions, defects, and/or an incorrect growing process. GID is an extremely accurate method for assessing surfaces that are the crucial interfaces for light collection and extraction. SEM is a powerful tool at a different level; the morphology of surface and particles can be studied at micro and nanometric scale. By energy dispersion spectrometry, qualitative and quantitative evaluation of the compounds can be carried out. In case of the necessity of atomic resolution, TEM offers the possibility of detecting lattice distortion, inclusions, and possible modification of the crystal cell.

Structural analysis and quality control are fundamental in both theoretical research and the production process. Except for conditions in which a specific characteristic of the tests is needed (time, non-invasiveness, resolution, etc.), a combination of the presented methodologies is preferred, aiming at a complete characterization of the anisotropic crystals.

Author Contributions: Conceptualization, N.P., L.S., D.R., F.D. and L.M.; methodology, N.P., L.S., D.R. and L.M., G.B., P.M.; software, L.M., P.P.N.; validation, L.S., L.M. and.; formal analysis, L.M., D.N., G.B., and F.D.; investigation, L.S., L.M., D.R.; resources, L.M., P.M., G.B., D.N.; data curation, L.S., G.B., P.M., D.R. and L.M.; writing-original draft preparation, L.M.; writing—review and editing, N.P., L.S., F.D. and L.M.; visualization, G.B. and P.M.; supervision, L.M. and D.R.; project administration, L.M. and F.D.; funding acquisition, N.P., L.S. and F.D.

Funding: This research was partially funded by the European Research Council under the COST action TD-1401 "FAST-Fast Advanced Scintillation Timing" and by the research grant of the Università Politecnica delle Marche: Progetto Strategico di Ateneo 2017: "Scintillating crystals: an interdisciplinary, applications-oriented approach aimed to the scientific knowledge and process control for application concerning life-quality improvement."

Acknowledgments: These research topics are within the scope of CERN R\&D Experiment 18 "Crystal Clear Collaboration" and the PANDA Collaboration at GSI-Darmstadt.

Conflicts of Interest: The authors declare no conflict of interest. The funders had no role in the design of the study; in the collection, analyses, or interpretation of data; in the writing of the manuscript; or in the decision to publish the results.

\section{Abbreviations}

The following abbreviations are used in this manuscript:

$\begin{array}{ll}\text { DICEA } & \text { Dipartimento di Ingegneria Civile, Edile e Architettura } \\ \text { DIISM } & \text { Dipartimento di Ingegneria Meccanica e Scienze Matematiche } \\ \text { SIMAU } & \text { Dipartimento di Scienze e Ingegneria della Materia, Ambiente e Urbanistica } \\ \text { ICRYS } & \text { Centro Interdipartimentale di Ricerca e Analisi Globale dei Cristalli } \\ \text { CERN } & \text { European Center for Nuclear Research, Geneva }(\mathrm{CH}) \\ \text { PANDA } & \text { Proton Annihilation in Darmstadt, GSI, Darmstadt (D) } \\ \text { CMS } & \text { Compact Muon Solenoid }\end{array}$

\section{References}

1. Lecoq, P. Development of new scintillators for medical applications. Nucl. Instrum. Methods Phys. Res. Sect. A Accel. Spectrom. Detect. Assoc. Equip. 2016, 809, 130-139. [CrossRef] 
2. Brudanin, V.B.; Gundorin, N.A.; Filossofov, D.V.; Nemtchenok, I.B.; Smolnikov, A.A.; Vasiliev, S.I.; Bregadze, V.I. Element-loaded organic scintillators for neutron and neutrino physics. In The Identification Dark Matter, Proceedings of the 3 rd International Workshop, York, UK, 18-22 September 2011; World Scientific Publishing Co. Pte. Ltd.: Singapore, 2011; No. 6; pp. 6626-6634.

3. Zhu, R.Y. The next generation of crystal detectors. J. Phys. Conf. Ser. 2015, 587. [CrossRef]

4. Sidletskiy, O. Trends in Search for Bright Mixed Scintillators. Phys. Status Solidi Appl. Mater. Sci. 2018, 215, 1701034. [CrossRef]

5. Gundacker, S.; Auffray, E.; Pauwels, K.; Lecoq, P. Measurement of intrinsic rise times for various L(Y)SO and LuAG scintillators with a general study of prompt photons to achieve $10 \mathrm{ps}$ in TOF-PET. Phys. Med. Biol. 2016, 61. [CrossRef] [PubMed]

6. Lecoq, P. Pushing the Limits in Time-of-Flight PET Imaging. IEEE Trans. Radiat. Plasma Med. Sci. 2017, 1, 473-485. [CrossRef]

7. Scalise, L.; Rinaldi, D.; Davì, F.; Paone, N. Measurement of ultimate tensile strength and Young modulus in LYSO scintillating crystals. Nucl. Instrum. Methods Phys. Res. Sect. A Accel. Spectrom. Detect. Assoc. Equip. 2011, 654. [CrossRef]

8. Ishii, M.; Harada, K.; Kobayashi, M.; Usuki, Y.; Yazawa, T. Mechanical properties of PbWO4 scintillating crystals. Nucl. Instrum. Methods Phys. Res. Sect. A Accel. Spectrom. Detect. Assoc. Equip. 1996, 376, 203-207. [CrossRef]

9. Boca, G. The PANDA experiment: Physics goals and experimental setup. EPJ Web Conf. 2014, 72, 2. [CrossRef]

10. Donghia, R. The Mu2e experiment at Fermilab: Design and status. NUOVO Cim. 2017, 40, 1-7.

11. Kimble, T.; Chou, M.; Chai, B.H.T. Scintillation properties of LYSO crystals. In Proceedings of the 2002 IEEE Nuclear Science Symposium Conference Record, Norfolk, VA, USA, 10-16 November 2002; Volume 3, pp. 1434-1437.

12. Ding, D.Z.; Yang, J.H.; Ren, G.H.; Nikl, M.; Wang, S.; Wu, Y.T.; Mao, Z.Y. Effects of anisotropy on structural and optical characteristics of LYSO: CE crystal. Phys. Status Solidi Basic Res. 2014, 251, 1202-1211. [CrossRef]

13. Rinaldi, D.; Montalto, L.; Lebeau, M.; Mengucci, P. Influence of a Surface Finishing Method on Light Collection Behaviour of PWO Scintillator Crystals. Photonics 2018, 5, 47. [CrossRef]

14. Montalto, L.; Natali, P.P.; Daví, F.; Mengucci, P.; Paone, N.; Rinaldi, D. Characterization of a defective $\mathrm{PbWO}_{4}$ crystal cut along the a-c crystallographic plane: Structural assessment and a novel photoelastic stress analysis. J. Instrum. 2017, 12. [CrossRef]

15. Rinaldi, D.; Ciriaco, A.; Lebeau, M.; Paone, N. Quality control on pre-serial Bridgman production of $\mathrm{PbWO}_{4}$ scintillating crystals by means of photoelasticity. Nucl. Instrum. Methods Phys. Res. Sect. A Accel. Spectrom. Detect. Assoc. Equip. 2010, 615, 254-258. [CrossRef]

16. Dally, J.W.; Riley, W.F. Experimental Stress Analysis; Kogushka McGraw Hill: Toledo, OH, USA, 1966.

17. Coker, E.G.; Filon, L.N.G. A Treatise on Photo-Elasticity; Cambridge University Press: Cambridge, UK, 1931.

18. Ewing, J.A. Photo—Elasticity; Nature Publishing Group: London, UK, 1932.

19. Born, M.; Wolf, E. Principles of Optics: Electromagnetic Theory of Propagation, Interference and Diffraction of Light; Cambridge university press: Cambridge, UK, 1955.

20. Wahlstrom, E.E. Optical Crystallography; Wiley: Hoboken, New Jersey, USA, 1969.

21. Rinaldi, D.; Ietroni, P.P.; Davì, F. Isochromate fringes simulation by Cassini-like curves for photoelastic analysis of birefringent crystals. Nucl. Instrum. Methods Phys. Res. Sect. A Accel. Spectrom. Detect. Assoc. Equip. 2009, 603, 294-300. [CrossRef]

22. Daví, F. On the Bertin surfaces of photoelastic crystals. J. Opt. Soc. Am.A 2015, 32, 2323. [CrossRef] [PubMed]

23. Daví, F.; Rinaldi, D. Mechanical and Optical Properties of Anisotropic Single-Crystal Prisms. J. Elast. 2015, 120, 197-224. [CrossRef]

24. Rinaldi, D.; Daví, F.; Montalto, L. On the photoelastic constants and the Brewster law for stressed tetragonal crystals. Math. Methods Appl. Sci. 2018, 41, 3103-3116. [CrossRef]

25. Ciriaco, A.; Davi, F.; Lebeau, M.; Majni, G. PWO photo-elastic parameter calibration by laser-based polariscope. Nucl. Instrum. Methods Phys. Res. Sect. A Accel. Spectrom. Detect. Assoc. Equip. 2007, 570, 55-60. [CrossRef]

26. Natali, P.P.; montalto, L.; Davi, F.; Menqucci, P.; Paone, N.; Rinaldi, D. Theoretical and experimental evaluation of piezo-optic parameters and photoelastic constant in tetragonal PWO. Appl. Opt. 2018, 57, 730-737. [CrossRef]

27. Mytsyk, B.G.; Andrushchak, A.S.; Demyanyshyn, N.M.; Kost, Y.P.; Kityk, A.V.; Mandracci, P.; Schranz, W. Piezo-optic coefficients of MgO-doped LiNbO_3 crystals. Appl. Opt. 2009, 48, 1904-1911. [CrossRef] 
28. Solskii, I.M.; Mytsyk, B.G.; Kost, Y.P.; Andrushchak, A.S.; Demyanyshyn, N.M. Piezo-optic coefficients of CaWO4 crystals. Crystallogr. Rep. 2015, 60, 130-137.

29. Mytsyk, B.G.; Dem yanyshyn, N.M. Piezo-optic surfaces of lithium niobate crystals. Crystallogr. Rep. 2006, 51, 653-660. [CrossRef]

30. Natali, P.P.; Montalto, L.; Rinaldi, D.; Davi, F.; Paone, N.; Scalise, L. Noninvasive Inspection of Anisotropic Crystals: Innovative Photoelasticity-Based Methods. IEEE Trans. Nucl. Sci. 2018, 65, 2203-2207. [CrossRef]

31. Montalto, L.; Paone, N.; Scalise, L.; Rinaldi, D. A photoelastic measurement system for residual stress analysis in scintillating crystals by conoscopic imaging. Rev. Sci. Instrum. 2015, 86, 86. [CrossRef] [PubMed]

32. Montalto, L.; Paone, N.; Rinaldi, D.; Scalise, L. Inspection of birefringent media by photoelasticity: From diffuse light polariscope to laser conoscopic technique. Opt. Eng. 2015, 54. [CrossRef]

33. Montalto, L.; Rinaldi, D.; Scalise, L.; Paone, N.; Davì, F. Photoelastic sphenoscopic analysis of crystals. Rev. Sci. Instrum. 2016, 87. [CrossRef] [PubMed]

34. Klug, H.P.; Alexander, L.E. X-Ray Diffraction Procedures_For Polycrystalline and Amorphous Materials; Wiley: New York, NY, USA, 1974.

35. Nielsen, J.A.; McMorrow, D. Elements of Modern X-Ray Physics, 2011th ed.; J. Wiley \& Sons Ltd.: Hoboken, NJ, USA, 2011.

36. Cullity, B.D.; Stock, S.R. Elements of X-Ray Diffraction, 2014th ed.; Pearson Education Ltd.: Upper Saddle River, NJ, USA, 2014.

37. Goldstein, J.; Newbury, D.E.; Joy, D.C.; Lyman, C.E.; Echlin, P.; Lifshin, E.; Sawyer, L.; Michael, J. R Scanning Electron Microscopy and X-Ray Microanalysis; Springer: New York, NY, USA, 1992.

38. Hirsch, P.B. Electron Microscopy of Thin Crystals; Krieger Publishing Company: Malabar, FL, USA, 1977.

39. Rudolph, P.; Kiessling, F. The horizontal bridgman method. Cryst. Res. Technol. 1988, 23, 1207-1224. [CrossRef]

40. Mengucci, P.; Cristoforo, A.D.; Lebeau, M.; Majni, G.; Paone, N.; Pietroni, N.; Rinaldi, D. Surface quality inspection of $\mathrm{PbWO}_{4}$ crystals by grazing incidence X-ray diffraction. Nucl. Instrum. Methods Phys. Res. Sect. A Accel. Spectrom. Detect. Assoc. Equip. 2005, 537. [CrossRef]

41. Barucca, G.; Davı, F.; Lancioni, G.; Mengucci, P.; Montalto, L.; Natali, P.P.; Paone, N.; Rinaldi, D.; Scalise, L.; Erni, W. Precision resonance energy scans with the PANDA experiment at FAIR: Sensitivity study for width and line shape measurements of the X(3872). Eur. Phys. J. A 2019, 55. [CrossRef]

42. David, S.; Georgiou, M.; Fysikopoulos, E.; Loudos, G. Evaluation of a SiPM array coupled to a Gd3Al2Ga3O12:Ce (GAGG:Ce) discrete scintillator. Phys. Med. 2015, 31, 763-766. [CrossRef]

43. Pizzichemi, M. Positron Emission Tomography: State of the art and future developments. J. Instrum. 2016, 11. [CrossRef]

44. Auffrayc, E.; Bel Hadjc, F.B.M.; Cortinovisa, D.; Doroudc, K.; Garuttib, E.; Lecoqc, P.; Liud, Z.; Martinezd, R.; Paganonid, M.; Pizzichemid, M.; et al. Characterization studies of silicon photomultipliers and crystals matrices for a novel time of flight PET detector. J. Instrum. 2015, 10, P06009. [CrossRef]

45. Menguccia, P.; Andréb, G.; Auffrayc, E.; Baruccaa, G.; Cecchi, C.; Chipauxe, R.; Cousson, A.; Davì, F.; Di Vara, N.; Rinaldia1, D.; et al. Structural, Mechanical and light yield characterisation of heat treated LYSO:Ce single crystals for medical imaging applications. Nucl. Instrum. Methods Phys. Res. Sect. A Accel. Spectrom. Detect. Assoc. Equip. 2015, 785, 110-116. [CrossRef]

46. Davi, F.; Rinaldi, D. Elastic moduli and optical properties of LYSO crystals: Theory and experiments. IEEE Trans. Nucl. Sci. 2012, 59. [CrossRef]

47. Trusova, E.; Vaitkevičius, A.; Tratsiak, Y.; Korjik, M.; Mengucci, P.; Rinaldi, D.; Montalto, L.; Marciulionyte, V.; Tamulaitis, G. Barium and lithium silicate glass ceramics doped with rare earth ions for white LEDs. Opt. Mater. Amst. 2018, 84, 459-465. [CrossRef]

48. Leutz, H. Scintillating fibres. Nucl. Instrum. Methods Phys. Res. Sect. A Accel. Spectrom. Detect. Assoc. Equip. 1995, 364, 422-448. [CrossRef]

49. Diehl, S.; Novotny, R.; Aubry, N.; Faraj, S.; Ferro, G. Development and Characterization of Inorganic Scintillating Fibers Made of LuAG:Ce and LYSO:Ce. IEEE Trans. Nucl. Sci. 2014, 61. [CrossRef]

(C) 2019 by the authors. Licensee MDPI, Basel, Switzerland. This article is an open access article distributed under the terms and conditions of the Creative Commons Attribution (CC BY) license (http://creativecommons.org/licenses/by/4.0/). 